\title{
Vorhofflimmern: Wie effektiv und sicher sind die direkten oralen Antikoagulanzien zur Prävention von Schlaganfällen?
}

\author{
Stefan H. Hohnloser, Máté Vámos, Hans-Christoph Diener
}

\begin{abstract}
Vorhofflimmern ist die in Praxis und Klinik am häufigsten diagnostizierte Herzrhythmusstörung. Es erhöht vor allem das Risiko für thromboembolische Komplikationen. Therapiestandard zur Prävention von Schlaganfällen bei diesen Patienten waren bislang Vitamin-K-Antagonisten. Die neuen direkten oralen Antikoagulanzien haben ihnen gegenüber jedoch hinsichtlich der Anwendung und Wirksamkeit entscheidende Vorteile.
\end{abstract}

Risiko Vorhofflimmern | Das Risiko für einen ischämischen Schlaganfall liegt bei Patienten mit Vorhofflimmern um das 4- bis 5-Fache höher als bei Sinus-rhythmischen Patienten [1]. Thromboembolische Komplikationen treten bei allen Formen von Vorhofflimmern auf. Ihre Inzidenz ist bei paroxysmalem, persistierendem und permanentem Vorhofflimmern ähnlich [2].

Vorhofflimmern und Schlaganfall | 20-25\% aller ischämischen Schlaganfälle sind auf Vorhofflimmern zurückzuführen. Dies verdeutlicht die enorme Bedeutung dieser Rhythmusstörung als signifikanter Risikofaktor für kardioembolische Schlaganfälle.

Der Verlauf von Schlaganfällen, die mit Vorhofflimmern assoziiert sind, ist wesentlich schwerer als von Schlaganfällen anderer Ursache.

Das belegen mehrere Studien:

- Marini et al. [3]:

- Die 30-Tage-Mortalität bei Vorhofflimmernassoziierten Schlaganfällen betrug 33\%, aber nur $16 \%$ bei Schlaganfällen anderer Ursache.

- Nach einem Jahr waren 50\% der Patienten mit Vorhofflimmern-bedingten Schlaganfällen gestorben, aber nur $27 \%$ derjenigen ohne Vorhofflimmern.

- Bei den überlebenden Patienten waren die funktionellen Ausfälle nach Vorhofflimmernassoziierten Schlaganfällen wesentlich gravierender als nach solchen anderer Ursache

- Dulli et al. [4]:

- Kortikale Defizite (z.B. Aphasie), schwere Paresen der Extremitäten, Bewusstseinsstörungen und Bettlägerigkeit waren bei Patienten mit Vorhofflimmern signifikant häufiger zu verzeichnen [4].

- Lamassa et al. [5]

- funktionellen Defizite (gemessen anhand des modifizierten Rankin-Score) waren 1,5-fach höher bei Patienten mit Vorhofflimmern-bedingten Schlaganfällen im Vergleich zu Patienten mit Ereignissen anderer Genese.
Rezidivrisiko | Bei Patienten, die bereits einen Schlaganfall oder eine TIA erlitten haben, ist das Rezidivrisiko hoch. In einer Metaanalyse [6] fand sich eine durchschnittliche Schlaganfallsrate von $13 \%$ pro Jahr bei Vorhofflimmern-Patienten mit stattgehabten Schlaganfällen. Bei Patienten ohne eine solche Anamnese lag die Rate bei 4,1\%.

Patienten mit Vorhofflimmern müssen möglichst wirkungsvoll vor thrombembolischen Ereignissen geschützt werden - insbesondere vor Schlaganfällen.

Schlaganfallprävention mit VKA | Vitamin-K-Antagonisten (VKA), namentlich Warfarin, wurden vor über 60 Jahren zur Prävention von tiefen Beinvenen-Thrombosen und Schlaganfällen in die Klinik eingeführt. In den vergangenen 25 Jahren sind insgesamt 6 Placebo-kontrollierte klinische Studien zur Prävention von thrombembolischen Ereignissen bei Patienten mit Vorhofflimmern durchgeführt worden. In einer Metaanalyse [7] dieser Untersuchungen, die insgesamt Daten von 2900 Patienten miteinbezog, zeigte sich:

- Die Therapie mit Warfarin war mit einer 64\%igen Reduktion (95\%-Konfidenzintervall [KI] 49-74\%) des Risikos für einen Schlaganfall im Vergleich zu Plazebo assoziiert.

- Absolute Risikoreduktion: 2,9\% pro Jahr

- Placebogruppe $4,5 \%$

- VKA-Gruppe 1,6\% pro Jahr.

- Im Vergleich zu einer Plättchen-Hemmung (4876 Patienten) betrug die Risikoreduktion zugunsten der VKA $37 \%$ (95\%-KI 23-48\%).

Somit ist die Effektivität einer sachgerechten Antikoagulation mit VKA zu Prävention von ischämischen Schlaganfällen bei Vorhofflimmern gut belegt.

ASS zur Schlaganfallprävention | Acetylsalicylsäure (ASS) hat - wenn überhaupt - nur eine geringe präventive Wirkung bezüglich thrombembolischer Ereignisse bei Vorhofflimmern. In der 
bereits angesprochenen Metaanalyse [7], die alle 7 dementsprechenden kontrollierten Studien mit einbezog, zeigte sich gegenüber Placebo lediglich eine 19\%ige Risikoreduktion (95\%-KI - 1 bis 35\%) für Schlaganfälle. Dies bestätigte auch eine randomisierte Studie an 973 Vorhofflimmern-Patienten im Alter von über 75 Jahren - sie waren mit VKA oder ASS behandelt worden [8]. Die VKA-Therapie reduzierte Schlaganfälle im Vergleich zur ASSTherapie hochsignifikant. Ebenso wichtig war jedoch die Analyse der Blutungsereignisse in dieser Studie: Für schwerwiegende Blutungen (inklusive intrakranieller Blutungen) zeigte sich kein signifikanter Unterschied zwischen der VKA- und der ASS-Gruppe, d.h. die Thrombozyten-Aggregationshemmung war mit einem ähnlichen Blutungsrisiko vergesellschaftet wie eine Antikoagulation mit VKA.

Die neuesten Therapierichtlinien zur Behandlung von Vorhofflimmern empfehlen die ASS-Therapie nicht mehr zur Schlaganfallprävention [9].

\section{Limitationen der Vitamin-K-Antagonisten}

Wirkung I VKA hemmen die Synthese der Gerinnungsfaktoren II, VII, IX und X, und werden über den Cytochrom-p450-Enzym-Komplex metabolisiert [10, 11]. Aus diesem Grund existieren zahlreiche Interaktionen mit anderen Medikamenten - nicht nur mit solchen, die das Gerinnungssystem beeinflussen. Die Effekte der VKA hängen daneben auch ab von

- der Leberfunktion,

- genetischen Faktoren,

- Alkoholgenuss,

- Nahrungsmitteln und

- vor allem von der Patientencompliance [10].

Ziel-INR-Bereich eng I VKA haben ein sehr enges therapeutisches Fenster in einem INR-Bereich zwischen 2,0 und 3,0:
- INR-Werte<2,0: unzureichende antithrombotische Effektivität und konsekutiv erhöhtes Risiko für einen ischämischen Schlaganfall

- INR-Werte>4,0: Risiko für Blutungen erhöht, insbesondere für intrakranielle Blutungen $[12,13]$. Bei vielen Patienten erfordert eine suffiziente INREinstellung unter VKA-Therapie zahlreiche Kontrolluntersuchungen - oft über lange Zeiträume. Sowohl Ärzte als auch Patienten sind deshalb allzu oft zurückhaltend bezüglich dieser Therapiemodalität. Daneben brechen viele Patienten eine VKA-Therapie häufig ab. Daten aus dem schwedischen Schlaganfall-Register belegen, dass mehr als die Hälfte der Patienten nach überstandenem Schlaganfall, die unter VKA-Therapie aus der Klinik entlassen werden, innerhalb von nur 2 Jahren die Antikoagulationstherapie beendeten [14].

Nutzen und Risiko abwägen | Die Prävention von ischämischen Schlaganfällen bei Vorhofflimmern muss ein vernünftiges Nutzen-Risiko-Verhältnis aufweisen. Auf der Risikoseite stehen vor allem Blutungen, insbesondere intrakranielle Blutungen.

Während Patienten in der Regel vor allem den Schlaganfall fürchten, fürchten Ärzte in vielen Fällen vor allem die Blutungskomplikationen.

Aus all diesen Gründen wird die Antikoagulationstherapie bei Vorhofflimmern-Patienten noch immer zu wenig durchgeführt:

- Bis zu 40\% der Hochrisiko-Patienten (Zustand nach TIA oder Schlaganfall) erhalten keine Antikoagulationstherapie zur sekundären Schlaganfallprävention [15].

- 60-80\% der Behandlungsfälle (d.h. Patienten ohne Kontraindikationen für eine solche Behandlung) erhalten eine Antikoagulationstherapie - mindestens ein Fünftel bis ein Drittel der Patienten mit Vorhofflimmern jedoch immer noch nicht $[16,17]$.

Leider gilt dies gerade auch für die Patienten mit hohem Schlaganfallrisiko. Aufgrund der Schwie-

Tab.1 Pharmakologie der neuen direkten oralen Antikoagulanzien im Vergleich zu den Vitamin-K-Antagonisten.

\begin{tabular}{|c|c|c|c|c|c|}
\hline & Apixaban & Dabigatran & Edoxaban & Rivaroxaban & Warfarin \\
\hline Wirkmechanismus & $\begin{array}{l}\text { Direkter Faktor-Xa- } \\
\text { Inhibitor }\end{array}$ & $\begin{array}{l}\text { Direkter Thrombin- } \\
\text { Inhibitor }\end{array}$ & $\begin{array}{l}\text { Direkter Faktor-Xa- } \\
\text { Inhibitor }\end{array}$ & $\begin{array}{l}\text { Direkter Faktor-Xa- } \\
\text { Inhibitor }\end{array}$ & $\begin{array}{l}\text { Inhibitor der Vitamin-K- } \\
\text { abhängigen Faktoren }\end{array}$ \\
\hline $\begin{array}{l}\text { Orale } \\
\text { Bioverfügbarkeit }\end{array}$ & $\sim 50 \%$ & $\sim 6,5 \%$ & $62 \%$ & $80-100 \%$ & $>95 \%$ \\
\hline Pro-Drug & Nein & Ja & Nein & Nein & Nein \\
\hline Effekt von Nahrung & Nein & Nein & Nein & $\begin{array}{l}\text { Ja (muss mit Nahrung } \\
\text { genommen werden) }\end{array}$ & $\begin{array}{l}\text { Ja (Vitamin-K-reiche } \\
\text { Nahrungsmittel) }\end{array}$ \\
\hline Renale Clearance & $\sim 27 \%$ & $85 \%$ & $49 \%$ & $\sim 33 \%$ & 0 \\
\hline $\mathrm{T}_{\max }$ & $3-4 h$ & $0.5-2 h$ & $1-2 h$ & $2-4 h$ & 3-5 Tage \\
\hline Halbwertszeit $\left(t_{1 / 2}\right)$ & $12 \mathrm{~h}$ & $12-17 \mathrm{~h}$ & $6-11 \mathrm{~h}$ & $\begin{array}{l}\text { 5-9h (jüngere Ptn) } \\
\text { 11-13 h (ältere) }\end{array}$ & $40 \mathrm{~h}$ \\
\hline
\end{tabular}


rigkeiten bei der Einstellung auf eine INR zwischen 2,0 und 3,0 muss außerdem davon ausgegangen werden, dass die meisten Patienten mit ihren INR-Werten lediglich etwa 50-60\% der Zeit in diesem therapeutischen Bereich liegen („time in therapeutic range, TTR“) $[18,19]$.

Die Limitationen der VKA-Therapie machen die Notwendigkeit für effektivere und sicherere Antikoagulationsmedikamente sehr deutlich.

\section{Neue direkte orale Antikoagulanzien}

Pharmakologie | In den letzten 10 Jahren sind insgesamt vier neue orale Antikoagulanzien für die Prävention von thrombembolischen Komplikationen bei Patienten mit Vorhofflimmern entwickelt worden. Diese hemmen dosisabhängig und selektiv entweder

- den Faktor II, Thrombin (Dabigatran) oder

- den Faktor Xa (Rivaroxaban, Apixaban, Edoxaban).

Wegen dieser selektiven Wirkung auf jeweils nur einen spezifischen Gerinnungsfaktor werden diese Substanzen auch als direkte orale Antikoagulantien (DOAC) bezeichnet. Alle Substanzen weisen neben dieser selektiven Wirkung zahlreiche andere pharmakologische und pharmakodynamische Vorteile gegenüber den VKA auf $(\bullet$ Tab. 1):

- kurze Halbwertszeit mit schnellem Wirkungseintritt und schnellem Abklingen der Wirkung (im Bereich von Stunden),

- keine Wechselwirkungen mit Nahrungsmitteln,

- lediglich einzelne Wechselwirkungen mit anderen Medikamenten sowie
- kein regelmäßiges Monitoring der blutverdünnenden Wirkung nötig

- bei fixen Tagesdosierungen weitgehend unabhängig vom Körpergewicht.

Aufgrund dieser Eigenschaften sind die DOAC wesentlich einfacher zu handhaben als die VKA.

Cave DOAC werden zu unterschiedlichen Anteilen renal eliminiert. Deshalb in allen Fällen regelmäßig die Nierenfunktion kontrollieren: mindestens jährlich, bei interkurrenten Erkrankungen aber häufiger.

Vergleich mit VKA | Für alle DOAC liegen die Ergebnisse großer randomisierter klinischer Studien vor, in denen sie mit Warfarin als Prototyp der VKA verglichen wurden $(\checkmark$ Tab. 2) [20-23]. Alle Studien verwendeten den denselben Effektivitätsendpunkt

- ischämischer Schlaganfall und

- systemische Embolie

sowie denselben Sicherheitsendpunkt

- schwerwiegende Blutungen.

Für diese Endpunkte wurden die entsprechenden Patientenzahlen in jeder einzelnen Studie berechnet. Bei allen Studien handelt es sich um sehr große Untersuchungen - zwischen 14000 und 21000 Patienten wurden eingeschlossen. Somit gehört das Prinzip der direkten oralen Antikoagulation zu den am besten untersuchten Therapieprinzipien der modernen Kardiologie und Neurologie.

Für die Indikation Schlaganfallprävention bei nicht-valvulärem Vorhofflimmern sind in Deutschland bislang Dabigatran, Rivaroxaban und Apixaban zugelassen.

Tab. 2 Kenndaten der großen Zulassungsstudien der direkten oralen Antikoagulantien. $\mathrm{CrCl}=$ Kreatinin-Clearance

\begin{tabular}{|c|c|c|c|c|c|c|}
\hline Studie & Antikoagulans & Einschlusskriterien & Vergleich & $\begin{array}{l}\text { Primärer } \\
\text { Endpunkt }\end{array}$ & $\begin{array}{l}\text { Primärer } \\
\text { Sicherheitsend- } \\
\text { punkt }\end{array}$ & $\begin{array}{l}\text { Ausschlusskriterium } \\
\text { bzgl. Nierenfunktion }\end{array}$ \\
\hline $\begin{array}{l}\text { RELY } \\
N=18113\end{array}$ & $\begin{array}{l}\text { Dabigatran } 2 \times \text { tgl. } \\
\text { - } 110 \mathrm{mg} \text { oder } \\
-150 \mathrm{mg}\end{array}$ & $\begin{array}{l}\text { Vorhofflimmern und } \\
\text { Rind. ein vaskulärer } \\
\text { Risikofaktor }\end{array}$ & Warfarin & $\begin{array}{l}\text { - Schlaganfall } \\
\text { - Embolie }\end{array}$ & Schwere Blutung & $\mathrm{CrCl}<30 \mathrm{ml} / \mathrm{min}$ \\
\hline $\begin{array}{l}\text { ROCKET AF } \\
N=14264\end{array}$ & $\begin{array}{l}\text { Rivaroxaban } 1 \times \text { tgl } \\
\text { - } 20 \mathrm{mg} \\
\text { - } 15 \mathrm{mg} \text { bei } \mathrm{CrCL} \\
\quad 30-49 \mathrm{ml} / \mathrm{min}\end{array}$ & $\begin{array}{l}\text { VHF und } \\
\text { Risikofaktoren }\end{array}$ & Warfarin & $\begin{array}{l}\text { - Schlaganfall } \\
\text { Embolie }\end{array}$ & Schwere Blutung & $\mathrm{CrCl}<30 \mathrm{ml} / \mathrm{min}$ \\
\hline $\begin{array}{l}\text { ARISTOTLE } \\
N=18201\end{array}$ & $\begin{array}{l}\text { Apixaban } 2 \times \text { tgl. } \\
\text { - } 5 \mathrm{mg} \\
\text { - } 2,5 \mathrm{mg}\end{array}$ & $\begin{array}{l}\text { Vorhofflimmern und } \\
\text { Rind. ein vaskulärer } \\
\text { Risikofaktor }\end{array}$ & Warfarin & $\begin{array}{l}\text { - Schlaganfall } \\
\text { - Embolie }\end{array}$ & Schwere Blutung & $\mathrm{CrCl}<25 \mathrm{ml} / \mathrm{min}$ \\
\hline $\begin{array}{l}\text { AVERROES } \\
N=5599\end{array}$ & $\begin{array}{l}\text { Apixaban } 2 \times \text { tgl. } \\
\text { - } 5 \mathrm{mg} \\
-2,5 \mathrm{mg}\end{array}$ & $\begin{array}{l}\text { Vorhofflimmern } \\
\text { Vitamin-K-Antago- } \\
\text { nisten ungeeignet }\end{array}$ & ASS & $\begin{array}{l}\text { - Schlaganfall } \\
\text { - Embolie }\end{array}$ & Schwere Blutung & $\mathrm{CrCl}<25 \mathrm{ml} / \mathrm{min}$ \\
\hline $\begin{array}{l}\text { Engage AF } \\
- \text { TIMI } 48 \\
N=21105\end{array}$ & $\begin{array}{l}\text { Edoxaban } 1 \times \text { tgl. } \\
\text { - } 60 \mathrm{mg} \text { oder } \\
\text { (Dosistitration erlaubt) }\end{array}$ & $\begin{array}{l}\text { Vorhofflimmern und } \\
\text { Risikofaktoren }\end{array}$ & Warfarin & $\begin{array}{l}\text { - Schlaganfall } \\
\text { - Embolie }\end{array}$ & Schwere Blutung & $\mathrm{CrCl}<30 \mathrm{ml} / \mathrm{min}$ \\
\hline
\end{tabular}




\begin{tabular}{|c|c|c|c|c|c|}
\hline & $\begin{array}{c}\text { DOAC } \\
\text { (Ereignisse) }\end{array}$ & $\begin{array}{l}\text { Warfarin } \\
\text { (Ereignisse) }\end{array}$ & & $\begin{array}{c}\text { RR } \\
(95 \%-K I)\end{array}$ & P-Wert \\
\hline RE-LY & $134 / 6076$ & 199/6022 & - - & $0,66(0,53-0,82)$ & 0,0001 \\
\hline ROCKET AF & $269 / 7081$ & $306 / 7090$ & - & $0,88(0,75-1,03)$ & 0,12 \\
\hline ARISTOTLE & $212 / 9120$ & $265 / 9081$ & - & $0,80(0,67-0,95)$ & 0,012 \\
\hline ENGAGE AF-TIMI 48 & 296/7035 & $337 / 7036$ & & $0,88(0,75-1,02)$ & 0,10 \\
\hline \multirow[t]{2}{*}{ Kombiniert (random) } & $911 / 29312$ & $1107 / 29229$ & & $0,81(0,73-0,91)$ & $<0,0001$ \\
\hline & & 0,5 & $\stackrel{\longleftarrow}{\longleftarrow}$ Bevorzugung DOAC & $\underset{\text { zugung Warfarin }}{\longrightarrow}$ & 2,0 \\
\hline
\end{tabular}

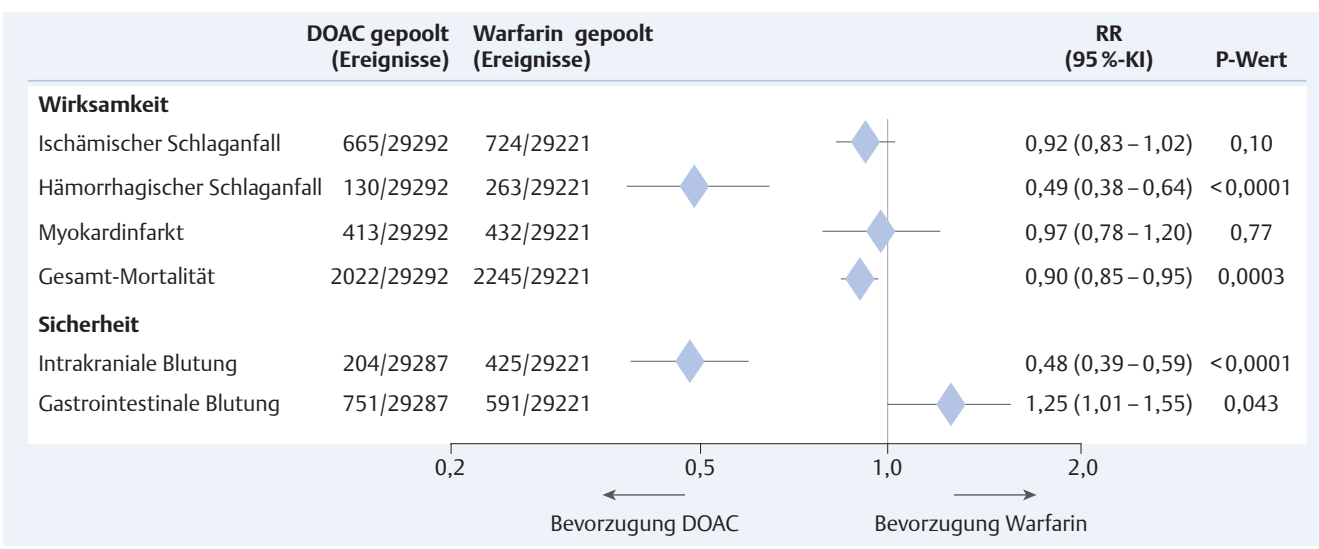

Wirksamkeit im Vergleich zu VKA | Betrachtet man die Datenlage der 4 großen Studien, sind alle DOAC mindestens so effektiv und sicher wie VKA, wenn nicht sogar signifikant überlegen [20-23]. In einer kürzlich publizierten systematischen Metaanalyse aller 4 Warfarin-kontrollierten $\mathrm{Zu}$ lassungsstudien der DOAC lässt sich die primäre Effektivität und Sicherheit in der Gesamtheit der Studienpopulationen und in wichtigen spezifischen Patientenuntergruppen beurteilen [24]. Von insgesamt 71683 Patienten waren 42411 mit einem DOAC und 29272 mit Warfarin behandelt worden.

- Die DOAC-Therapie führte im Vergleich zu einer Dosis-angepassten optimierten VKA-Behandlung zu einer hochsignifikanten Risikosenkung für einen Schlaganfall oder eine periphere Embolie bei nicht-valvulärem Vorhofflimmern (relative Risikoreduktion bei der Betrachtung aller Studien 19\%) ( Abb. 1).

Dieses Ergebnis ist konsistent mit den Beobachtungen der einzelnen Studien - hier lagen die relative Risikoreduktionen zwischen 12 und 34\% Sämtliche Endpunktereignisse in allen Studien wurden von kardiologischen und neurologischen Experten, die die jeweilige Therapiezuteilung der Patienten nicht kannten, hinsichtlich der genauen Diagnose klassifiziert. Wichtig ist daher, dass es vor allem hämorrhagische Schlaganfälle sind, die unter DOAC-Therapie wesentlich seltener auftreten als unter VKA: Hier wird das Risiko um die Hälfte reduziert ( $\bullet$ Abb. 2 ). Da diese speziellen Schlaganfälle mit einer hohen Mortalität assoziiert sind, ist es absolut konsistent, dass die Gesamtsterblichkeit unter DOAC-Therapie hochsig- nifikant um 10\% im Vergleich zu den VKA gesenkt werden konnte.

Sicherheit im Vergleich zu VKA | Die gefürchtetste Komplikationen jeder Antikoagulationstherapie sind schwerwiegende Blutungen, insbesondere intrakranielle Blutungen. Vielfach wurde in der Vergangenheit daher bei Vorhofflimmern keine Antikoagulationstherapie durchgeführt $[25,26]$.

- Die Gabe eines DOAC war im Vergleich zu Warfarin mit einer relativen Risikominderung von $14 \%$ für schwere Blutungen assoziiert $(P=0,06)$

- Abb. 3 [24].

- Die größte Risikominderung in den einzelnen Studien war für Apixaban (29\%) sowie für Edoxaban $(20 \%)$ zu verzeichnen.

Wichtiger als die Inzidenz aller schwerwiegenden Blutungen ist die Häufigkeit intrakranieller Blutungen (Summe aus hämorrhagischen Schlaganfällen und subduralen Hämatomen).

- Hier war eine Risikominderung von 52\% zugunsten der DOAC zu verzeichnen ( Abb. 2).

Die Reduktion intrakranieller Blutungen war in den einzelnen Studien konsistent für alle DOAC. Die einzige spezifische Blutungskomplikation, die im Mittel häufiger unter Behandlung mit DOAC auftrat, waren gastrointestinale Blutungen. Hier scheinen jedoch Unterschiede zwischen den einzelnen DOAC zu bestehen.

Apixaban war als einziges der neuen Medikamente nicht mit einer Zunahme gastrointestinaler Blutungen im Vergleich zu Warfarin assoziiert.
Abb. 1 „Schlaganfall und periphere Embolie" als primärer Effektivitätsendpunkt in der Metaanalyse aus den großen Zulassungsstudien der direkten oralen Antikoagulanzien bei Patienten mit nicht-valvulärem Vorhofflimmern [24].

Abb. 2 Wichtige Effektivitätsund Sicherheitsendpunkte in der Metaanalyse aus den großen Zulassungsstudien der direkten oralen Antikoagulanzien bei Patienten mit nicht-valvulärem Vorhofflimmern [24]. 
Abb. 3 "Schwerwiegende Blutungen" als primärer Sicherheitsendpunkt in der Metaanalyse aus den großen Zulassungsstudien der direkten oralen Antikoagulantien bei Patienten mit nicht-valvulärem Vorhofflimmern [24].

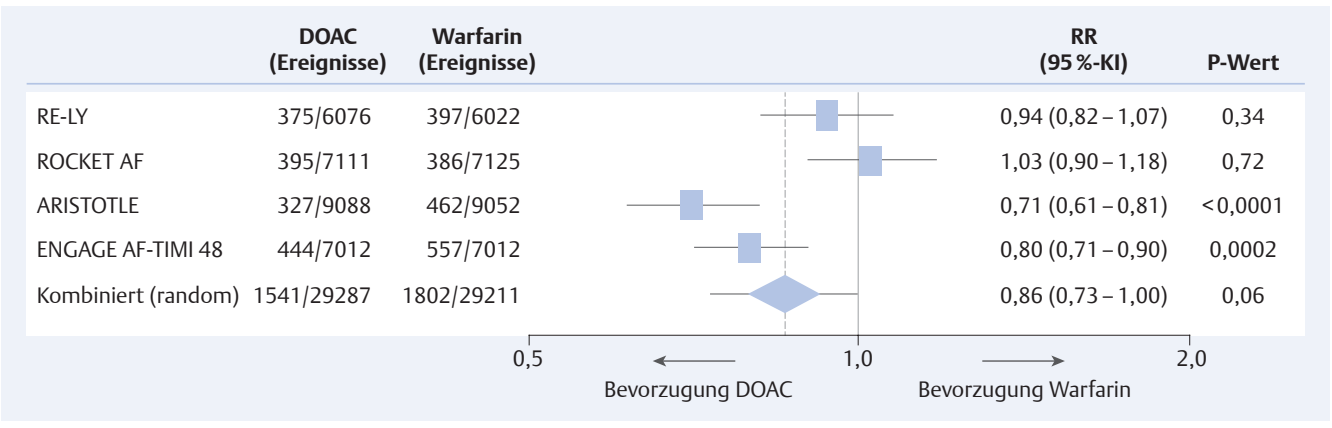

Dies ist bei entsprechender Anamnese daher differenzialtherapeutisch von Bedeutung.

Primäre vs. sekudäre Prävention | Das höchste Risiko für Vorhofflimmern-assoziierte Schlaganfälle haben Patienten, die bereits eine transitorisch ischämische Attacke oder einen Schlaganfall erlitten haben. In allen DOAC-Studien wurden solche Patienten eingeschlossen. Für Dabigatran, Rivaroxaban und Apixaban liegen bereits entsprechende Subanalysen vor [27-29]: Sie zeigen gerade bei sekundär-prophylaktisch behandelten Patienten eine deutliche Überlegenheit in puncto Effektivität und Sicherheit gegenüber den VKA.

Koronare Herzkrankheit | Für Patienten, die neben dem Vorhofflimmern eine begleitende koronare Herzkrankheit haben, liegen ebenfalls entsprechende Analyse zu Wirksamkeit und Sicherheit vor [30-32] - die Patienten profitieren von den neuen Medikamenten. Wie $>$ Abb. 2 zeigt, ist das Risiko für einen Herzinfarkt unter DOAC-Therapie im Vergleich zu den VKA nicht erhöht.

KHK und Plättchenhemmung | Ein ganz spezielles Problem ist bei KHK-Patienten oft die begleitende Plättchen-hemmende Therapie (ASS, P2Y12 Hemmer). Prinzipiell muss davon ausgegangen werden, dass eine kombinierte Behandlung mit Plättchenhemmern und oralen Antikoagulanzien die Blutungsgefahr natürlich erhöht. Die entsprechenden Analysen z.B. für Dabiga-
Das müssen Sie bei der Behandlung mit DOAC beachten:

- Die Wirkung tritt schnell ein (innerhalb von Stunden) - Eine überbrückende Heparingabe ist nicht notwendig.

- Die Wirkung klingt schnell ab.

- In den meisten Fällen ist vor elektiven Eingriffen keine überbrückende Heparingabe notwendig.

- Aufgrund der vorhersagbaren antikoagulatorischen Wirksamkeit ist kein regelhaftes Monitoring der Gerinnung nötig.

- Bei der Umstellung von nieder-molekularen Heparinen (LMWH) auf DOAC erfolgt die erste DOAC-Gabe zum Zeitpunkt der nächsten LMWH-Gabe.

- Nach einer VKA-Therapie wird die DOAC-Therapie begonnen, wenn INR $\leq 2,0$

- Es gibt nur sehr wenige Interaktionen der DOAC mit anderen Arzneimitteln.

- DOAC interagieren nicht mit Nahrungsmitteln.

- Nierenfunktion unter DOAC-Therapie mindestens einmal pro Jahr kontrollieren - bei interkurrenten Erkrankungen häufiger. tran [30] oder Apixaban [31] zeigen aber: Auch unter kombinierter Therapie ist die Blutungshäufigkeit unter DOAC niedriger als unter VKA. Die komplette Problematik der kombinierten Therapie bei verschiedenen Indikationen hier abzuhandeln, würde den Rahmen dieses Beitrags sprengen.

Niereninsuffizienz | Alle DOAC werden zu unterschiedlichen Anteilen renal eliminiert ( $\bullet$ Tab. 1). Für Dabigatran ist dieser Anteil am größten. Da bei vielen, gerade älteren Patienten mit Vorhofflimmern die Nierenfunktion eingeschränkt ist, muss sie sorgfältig beachtet werden. Aktuelle Therapierichtlinien [9] empfehlen deshalb mindestens jährliche Kontrolluntersuchungen der Nierenfunktion, bei interkurrenten Erkrankungen durchaus auch häufiger. Für alle DOAC gibt es für Patienten mit einer reduzierten Nierenfunktion reduzierte Dosisschemata, die auch in den entsprechenden Zulassungsstudien überprüft wurden.

Cave Ab einer Kreatinin-Clearance von $<30 \mathrm{ml} /$ min sollen jedoch keine DOACs mehr verwenden [9].

Kardioversion | Bei vielen Patienten mit symptomatischem Vorhofflimmern wird eine elektrische oder pharmakologische Konversion in den Sinusrhythmus angestrebt. Derartige Kardioversionen haben ein Risiko für thrombembolische Komplikationen (1-3\% der Fälle).

- Deshalb wird eine Antikoagulation für mindestens 3 Wochen vor und für 4 Wochen nach Kardioversion empfohlen [9].

Für Rivaroxaban liegt mittlerweile eine prospektive randomisierte Studie an mehr als 1500 Patienten vor, in der seine Effektivität und Sicherheit mit VKA vor und nach Kardioversion verglichen wurde [33]. Rivaroxaban war mit dem bisherigen Therapiestandard vergleichbar. Für Dabigatran liegt eine große Post-hoc-Analyse an 1270 Patienten aus der RE-LY Studie vor, die ebenfalls eine dem Warfarin vergleichbare Wirksamkeit und Sicherheit zeigen konnte [34]. Schließlich gibt es vergleichbare Daten auch für das Apixaban aus der entsprechenden großen Zulassungsstudie [35]. 
Akuter Schlaganfall | Erleidet ein Patient mit Vorhofflimmern einen ischämischen Schlaganfall, so ergibt sich die Frage nach eine Lysetherapie.

Eine orale Antikoagulation mit Vitamin-K-Antagonisten oder DOAC ist eine Kontraindikation für eine systemische Thrombolyse mit rt-PA bei Patienten mit akutem ischämischem Insult.

Liegt bei einem mit Vitamin-K-Antagonisten behandelten Patienten die INR bei Aufnahme unter 1,7 , kann allerdings die systemische Lyse durchgeführt werden [36]. Als Point-of-care-Test steht für Patienten unter Dabigatran-Behandlung die Messung der aPTT zur Verfügung. Ist diese normal, liegt bei Patienten mit normalen Nierenfunktionen die letzte Einnahme von Dabigatran mehr als 24 Stunden zurück und der Patient kann systemisch lysiert werden [37]. Einen ähnlichen Schnelltest gibt es für Apixaban und Rivaroxaban nicht. Bei Patienten mit distalen Verschlüssen der A. carotis interna oder mit proximalen Verschlüssen $\operatorname{der}$ A. cerebri media kann eine Thrombektomie mit einem Stent-Retriever auch bei wirksamer Antikoagulation durchgeführt werden.

Wann nach Schlaganfall beginnen? | Ebenso stellt sich oft die Frage, wann nach einem Schlaganfall mit einer DOAC-Therapie begonnen werden kann. Die European Society of Cardiology und die European Heart Rhythm Association empfehlen Folgendes [38]:

- Bei TIA am Tag nach der initialen Bildgebung beginnen.

- Bei leichtem Schlaganfall und geringem morphologischem Defizit in der zerebralen Bildgebung 3 Tage abwarten.

- Bei mittelschweren Schlaganfällen mindestens 6 Tage abwarten.

- Bei schweren Schlaganfällen 12-14 Tage abwarten.

- Bei mittelschweren und schweren Schlaganfällen muss vor Initiierung der oralen Antikoagulation eine Kontroll-Bildgebung erfolgen, um eine relevante hämorrhagische Transformation auszuschließen.

Diese Empfehlungen sind allerdings nicht Evidenz-basiert, da in den großen Studien Patienten mit akuten Schlaganfällen ausgeschlossen waren.

Entwicklung spezifischer Antidots | Wie bereits ausgeführt, ist die Inzidenz schwerwiegender Blutungskomplikationen, insbesondere von intrazerebralen Blutungen, unter DOAC-Therapie im Vergleich zu VKA deutlich geringer. Darüber hinaus wurde gezeigt, dass die Prognose von Patienten, die unter Apixaban bluteten, deutlich besser war als die von Patienten, die unter Warfarin bluteten [39]. Derzeit werden spezifische „reversal agents“ oder Antidots gegen ThrombinAntagonisten bzw. gegen Faktor-Xa-Hemmer entwickelt. In experimentellen Untersuchungen sowie in ersten klinischen Studien konnte dabei gezeigt werden, dass diese Antidots in der Lage sind, die antikoagularorische Wirkung der entsprechenden DOAC innerhalb von wenigen Minuten vollständig und langfristig aufzuheben. Die Verfügbarkeit derartiger Antidots wird die Sicherheit der Therapie mit DOAC noch weiter verbessern.

Mit der Zulassung des ersten derartigen Medikaments, des spezifischen Dabigatran-Antidots Idarucizumab [40], wird noch im Jahr 2015 gerechnet.

\section{Konsequenz für Klinik und Praxis}

- Die Entwicklung der direkten oralen Antikoagulanzien ist einer der größten medizinischen Fortschritte der letzten 10 Jahre.

- Im Vergleich zu den Vitamin-K-Antagonisten sind diese Medikamente ungleich einfacher anzuwenden und weisen eine bessere Wirksamkeit und eine höhere Sicherheit auf.

- Insbesondere die viel geringere Häufigkeit intrakranieller Blutungen machen die DOAC zu Medikamenten der ersten Wahl für die primäre und sekundäre Schlaganfallprävention bei nicht-valvulärem Vorhofflimmern.

\section{Interessenkonflikt}

S.H.H. gibt an, Honorare der folgenden Firmen für die Teilnahme an klinischen Studien, Mitarbeit in Advisory Boards und Vorträge erhalten zu haben: Bayer Healthcare, Boehringer Ingelheim, Bristol-Myers Squibb, Boston Scientific, Cardiome, Gilead, Johnson \& Johnson, Pfizer, Portola, Sanofi Aventis, Servier, St. Jude Medical und Zoll. M. V. gibt an, dass kein Interessenkonflikt besteht. H.C. D. gibt an, Honorae der folgenden Firmen für die Teilnahme an klinischen Studien, Mitarbeit in Advisory Boards und Vorträge erhalten zu haben: Abott, Allergan, AstraZeneca, Bayer Vital, Bristol-Myers-Squibb, Boehringe Ingelheim, CoAxia, Corimmun, Covidien, Daiichi-Sankyo, D-Pharm, Fresenius, GlaxoSmithKline, Janssen Cilag, Lilly, Lundbeck, Medtronic, MSD, MindFrame, Neurobiological Technologies, Novartis, Novo-Nordisk, Paion, Parke-Davis, Pfizer, Sanofi-Aventis, Schering, Servier, Solvay, Syngis, Tacrelis, Thrombogenics, Wyeth und Yamanouchi. Forschungsprojekte der Universitätsklinik für Neurologie in Essen wurden unterstützt von: AstraZeneca, GSK, Boehringer Ingelheim, Novartis, Janssen-Cilag und SanofiAventis.

\section{Literatur}

1 Wolf PA, Abbott RD, Kannel WB. Atrial fibrillation as an independent risk factor for stroke: The Framingham Study. Stroke. 1991; 22: 983-988

2 Hohnloser SH, Pajitnev D, Pogue J et al. Incidence of stroke in paroxysmal versus sustained atrial fibrillation in patients taking oral anticoagulation or combined antiplatelet therapy: an ACTIVE W Substudy. J Am Coll Cardiol 2007; 50: 2156-161

Vollständiges Literaturverzeichnis unter http://dx.doi.org/10.1055/s-0041-102000

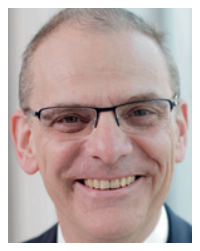

Prof. Dr. Stefan H. Hohnloser ist Leiter der Abteilung Klinische Elektrophysiologie an der Medizinischen Klinik III, Kardiologie der J. W. Goethe Universität Frankfurt Hohnloser@em.uni-frankfurt. de

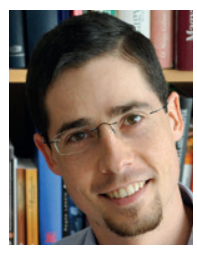

Dr. Maté Vámos

ist wissenschaftlicher Assistent in der Abteilung Klinische Elektrophysiologie, Medizinische Klinik III, Kardiologie der J. W. Goethe Universität Frankfurt

vamos.mate@gmail.com

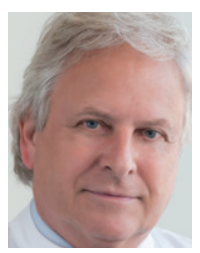

Prof. Dr.

Hans-Christoph Diener ist Direktor der Universitätsklinik für Neurologie in Essen mit dem Forschungsschwerpunkt Akuttherapie und Prophylaxe des Schlaganfalls hans.diener@uni-due.de

DOI 10.1055/s-0041-102000 Dtsch Med Wochenschr 2015; 140: 750-755

(c) Georg Thieme Verlag KG . Stuttgart · New York . ISSN 0012-0472 
3 Marini C, De Santis F, Sacco S et al. Contribution of atrial fibrillation to incidence and outcome of ischemic stroke: results from a population-based study. Stroke 2005; 36: 1115-1119

4 Dulli DA, Stanko H, Levine RL. Atrial fibrillation is associated with severe acute ischemic stroke. Neuroepidemiology 2003; 22: 118-123

5 Lamassa M, Di Carlo A, Pracucci G et al. Characteristics, outcome, and care of stroke associated with atrial fibrillation in Europe. Stroke 2001; 32: 392-398

6 Stroke Risk in Atrial Fibrillation Working Group. Independent predictors of stroke in patients with atrial fibrillation: a systematic review. Neurology 2007; 69: 546-554

7 Hart R, Benavente O, McBride R, Pearce L. Antithrombotic therapy to prevent stroke in patients with atrial fibrillation: a meta-analysis. Ann Intern Med 1999; 131 : 492-501

8 Mant J, Hobbs FDR, Fletcher $\mathrm{K}$ et al. Warfarin versus aspirin for stroke prevention in an elderly community population with atrial fibrillation (the Birmingham Atrial Fibrillation Treatment of the Aged Study, BAFTA): a randomised controlled trial. Lancet 2007; 370 : 493-503

9 Camm AJ, Lip GYH, De Caterina R et al. 2012 focused update of the ESC Guidelines for the management of atrial fibrillation. Europ Heart J 2012; 33: 2719-2749

10 Ansell J, Hirsh J, Hylek E et al. Pharmacology and management of the vitamin $\mathrm{K}$ antagonists: American College of Chest Physicians Evidence-Based Clinical Practice Guidelines (8th Edition). Chest 2008; 133 (Suppl. 6): 160S-1985

11 Turpie AG. New oral anticoagulants in atrial fibrillation. Eur Heart J 2008; 29: 155-165

12 Hylek E, Singer D. Risk factors for intracranial hemorrhage in outpatients taking warfarin. Ann Intern Med 1994; 120: 897-902

13 Oden A, Fahlen M, Hart RG. Optimal INR for prevention of stroke and death in atrial fibrillation: a critical appraisal. Thromb Res 2006; 117: 493-499

14 Glader EL, Sjolander M, Eriksson M, Lundberg M. Persistent use of secondary preventive drugs declines rapidly during the first 2 years after stroke. Stroke 2010; 41: 397-401

15 Ogilvie IM, Newton N, Welner SA et al. Underuse of oral anticoagulants in atrial fibrillation: a systematic review. Am J Med 2010; 123: 638-645

16 Kakkar AK, Mueller I, Bassandet JP et al. Risk profiles and antithrombotic treatment of patients newly diagnosed with atrial fibrillation at risk of stroke: Perspectives from the International, Observational, Prospective GARFIELD Registry. PLoS One 2013; 8: e63479

17 Meinertz T, Kirch W, Rosin L et al. Management of atrial fibrillation by primary care physicians in Germany: baseline results of the ATRIUM registry. Clin Res Cardiol 2011; 100: 897-905

18 Baker JWL, Cios DA, Sander SD, Coleman CI. MetaAnalysis to assess the quality of warfarin control in atrial fibrillation patients in the United States. J Manag Care Pharm 2009; 15: 2344-2252

19 Dlott JS, George RA, Huang X et al. National assessment of warfarin anticoagulation therapy for stroke prevention in atrial fibrillation. Circulation 2014; 129 : 1407-1414

20 Connolly SJ, Ezekowitz MD, Yusuf S et al. Dabigatran versus warfarin in patients with atrial fibrillation. $\mathrm{N}$ Engl | Med 2009; 361: 1139-1151

21 Granger C, Alexander J, McMurray J et al. Apixaban versus Warfarin in Patients with Atrial Fibrillation. N Engl J Med 2011; 365: 981-992.

22 Patel MR, Mahaffey KW, Garg | et al. Rivaroxaban versus warfarin in nonvalvular atrial fibrillation. N Engl J Med 2011; 365: 883-891.

23 Giugliano RP, Ruff CT, Braunwald E et al. Edoxaban versus warfarin in patients with atrial fibrillation. N Engl J Med 2013; 369: 2093-2104.
24 Ruff TR, Giugliano RP, Braunwald E et al. Comparison of the efficacy and safety of new oral anticoagulants with warfarin in patients with atrial fibrillation: a meta-analysis of randomised trials. Lancet 2014; 383: 955-962

25 Hylek E, D’Antonio J, Evans-Molina C et al. Translating the results of randomized trials into clinical practice. Stroke 2006; 37: 1075-1080

26 Lip GYH, Andreotti F, Fauchier L et al. Bleeding risk assessment and management in atrial fibrillation patients. Thromb Haemost 2011; 106: 997-911

27 Diener HC, Connolly SJ, Ezekowitz MD et al. Dabigatran compared with warfarin in patients with atrial fibrillation and previous transient ischaemic attack or stroke: a subgroup analysis of the RE-LY trial. Lancet Neurol 2010; 9: 1157-1163

28 Hankey GH, Patel MR, Stevens SR et al. Rivaroxaban compared with warfarin in patients with atrial fibrillation and previous stroke or transient ischaemic attack: a subgroup analysis of ROCKET AF. Lancet Neurol 2012; 11: 315-322

29 Easton JD, Lopes RD, Bahit MC et al. Apixaban compared with warfarin in patients with atrial fibrillation and previous stroke or transient ischaemic attack: a subgroup analysis of the ARISTOTLE trial. Lancet Neurol 2012; 11: 503-511

30 Hohnloser SH, Oldgren J, Yang S et al. Myocardial ischemic events in patients with atrial fibrillation treated with dabigatran or warfarin in the RE-LY (Randomized evaluation of long-term anticoagulation therapy) trial. Circulation 2012; 125: 669-676

31 Dans AL, Connolly SJ, Wallentin L et al. Concomitant Use of Antiplatelet Therapy with Dabigatran or Warfarin in the Randomized Evaluation of Long-Term Anticoagulation Therapy $\left(\right.$ RE-LY $\left.{ }^{\circledR}\right)$ Trial. Circulation 2013; 127: 634-640

32 Bahit MC, Lopes RD, Wojdyla DM et al. Apixaban in patients with atrial fibrillation and prior coronary artery disease: Insights from the ARISTOTLE trial. Int ] Cardiol 2013; 170: 215-220

33 Cappato R, Ezekowitz MD, Klein AL et al. Rivaroxaban versus Vitamin K Antagonists for Cardioversion in Atrial Fibrillation. Eur Heart ] 2014; 35: 3346-3355

34 Nagarakanti R, Ezekowitz MD, Oldgren J et al. Dabigatran versus warfarin in patients with atrial fibrillation. An analysis of patients undergoing cardioversion. Circulation 2011; 123: 131-136.

35 Flaker G, Lopes RD, Al-Khatib SM et al. Efficacy and safety of apixaban in patients after cardioversion for atrial fibrillation. Insights from the ARISTOTLE trial. J Am Coll Cardiol 2014; 63: 1082-1087.

36 Xian Y, Liang L, Smith EE et al. Risks of intracranial hemorrhage among patients with acute ischemic stroke receiving warfarin and treated with intravenous tissue plasminogen activator. JAMA 2012; 307: 2600-2608.

37 Diener HC, Foerch C, Riess H et al. Treatment of acute ischaemic stroke with thrombolysis or thrombectomy in patients receiving anti-thrombotic treatment. Lancet Neurol 2013; 12: 677-688.

38 Heidbuchel H, Verhamme P, Alings M et al. European Heart Rhythm Association Practical Guide on the use of new oral anticoagulants in patients with non-valvular atrial fibrillation. Europace 2013; 15: 625-651.

39 Hylek EM, Held C, Alexander JH et al. Major Bleeding in Patients with Atrial Fibrillation Receiving Apixaban or Warfarin in the ARISTOTLE Trial: Predictors, Characteristics, and Clinical Outcomes. J Am Coll Cardiol 2014; 63: 2141-2147

40 Schiele F, van Ryn J, Canada K et al. A specific antidote for dabigatran: functional and structural Characterization. Blood 2013; 121: 3554-3562 